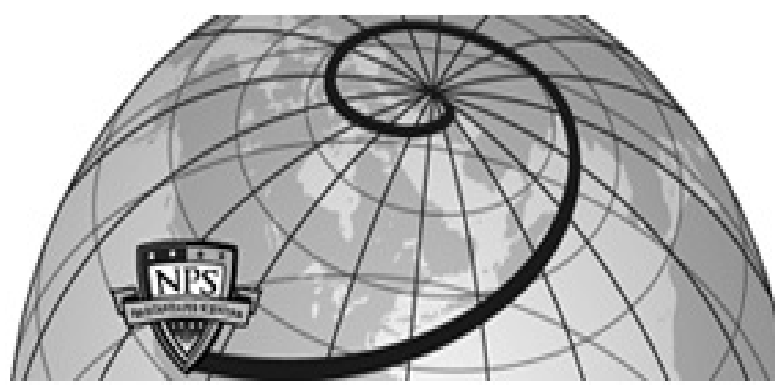

Calhoun: The NPS Institutional Archive DSpace Repository

Introduction of First Passage Time (FPT) Analysis for Software Reliability and Network Security

Ma, Zhanshan (Sam); Krings, Axel W.; Millar, Richard C.

https://hdl.handle.net/10945/43548

This publication is a work of the U.S. Government as defined in Title 17, United States Code, Section 101. Copyright protection is not available for this work in the United States.

Downloaded from NPS Archive: Calhoun

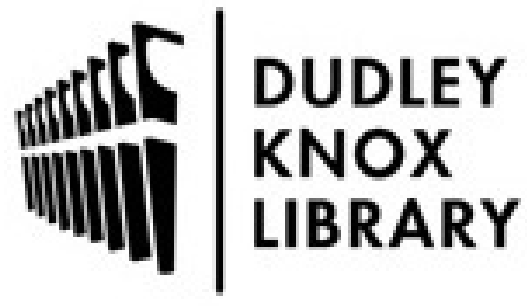

http://www.nps.edu/library
Calhoun is the Naval Postgraduate School's public access digital repository for research materials and institutional publications created by the NPS community. Calhoun is named for Professor of Mathematics Guy K. Calhoun, NPS's first appointed -- and published -- scholarly author.

Dudley Knox Library / Naval Postgraduate School 411 Dyer Road / 1 University Circle Monterey, California USA 93943 


\title{
Introduction of First Passage Time (FPT) Analysis for Software Reliability and Network Security
}

\author{
Zhanshan (Sam) Ma \\ Axel W. Krings \\ Richard C. Millar \\ $\underline{\text { ma@vandals.uidaho.edu }}$ \\ krings@uidaho.edu \\ rcmillar@nps.edu \\ Computer Science Department \\ University of Idaho, Moscow, ID 83844 \\ Department of Systems Engineering \\ Naval Postgraduate School
}

\begin{abstract}
The study of the First Passage Time (FPT) problem (also known as first passage problem, FPP) started more than a century ago, but its diverse applications in science and engineering mostly emerged in the last two to three decades. Assuming that $X(t)$ is a one-dimensional stochastic process, the First Passage Time is defined as the time $(T)$ when $X(t)$ first crosses a threshold. Engineering reliability is obviously a suitable application domain, and indeed applications such as optimal dam design in hydrology and analysis of structural failure in civil and mechanical engineering are typical examples. Although we envision that the FPT problem has great potential in network and software reliability, it should be more useful for network security and survivability because the approaches developed for the FPT problem are mostly analytical. The assumption for this inference is that in reliability analysis, experimental or historical data are often more readily available, which makes statistical approaches such as survival analysis more convenient and likely more realistic. In contrast, data is generally more difficult to obtain in security and survivability analyses, and analytical approaches can be leveraged to play more important roles. Furthermore, security and survivability often have to deal with malicious actions that may be driven by sophisticated cognition and behavioral processes, which are highly variable over time and very difficult to detect with short term data. If the behavior of an intruder can be characterized with some stochastic process such as Brownian motion, then the FPT approach may be applied to find the closedform solution of the probability density function (PDF) of the first passage time, which can be the time when the system breaks down or when the hacker is successful in compromising a network. In addition, the solutions to FPT depend on boundary and initial conditions of the corresponding partial differential equations, and they also describe the evolution of PDF over time. This may suggest that it is possible to model the behavior changes of an intruder over time and circumstances. Another advantage of FPT analysis is that it may help solve some non-Markov stochastic process problems in reliability analysis and survival analysis. In this article, we first briefly introduce the FPT problem with Brownian motion as an example, and then suggest its potential applications in software reliability and network security.
\end{abstract}

Permission to make digital or hard copies of all or part of this work for personal or classroom use is granted without fee provided that copies are not made or distributed for profit or commercial advantage and that copies bear this notice and the full citation on the first page. To copy otherwise, to republish, to post on servers or to redistribute to lists, requires prior specific permission and/or a fee.

The Fifth CSIIRW '2009, April 13-15, Oak Ridge National Lab, Oak Ridge, Tennessee, USA. Copyright $@ 2009$ ACM 978-1-60558-518-5... \$5.00

\section{Categories and Subject Descriptors}

C.2.4 [Distributed Systems]

General Terms: Reliability, Algorithms, Theory.

Keywords: First Passage Time (FPT); Mean First Passage Time (MFPT); Software Reliability, Network Security; Network Survivability; Intrusion Detection; Fokker-Planck Equation.

\section{INTRODUCTION}

Given a stochastic process $X(t)$ with $X(0)=0$, the first passage time (FPT) $T$ to the point $x=a>0$ is specified with

$$
T=\inf \{t: X(t)=a\} .
$$

In other words, FPT is the time when the stochastic process first crosses a threshold $a$. The FPT problem usually refers to finding the probability density function (PDF) of $T$ (Grimmet \& Stirzaker 1994, Ding \& Rangarajan 2004).

The history of the FPT problem can be traced at least back to 1900 (Bachelier 1900). It is a field that involves partial differential equations, stochastic differential equations, probability theory and stochastic processes. The most important application fields have been statistical physics and structure analysis (in civil and mechanic engineering). In physical science, the FPT problem is extensively studied in statistical physics and physical chemistry, especially in the study of diffusion in fluctuating media such as random walks in dynamically disordered systems (Revelli et al. 2002, Kulkarni \& Tzenova 2002, Rangarajan \& Ding 2000). In civil and mechanical engineering, it has been applied to study structures subjected to dynamically stochastic loading, which is essentially the reliability and fault detection problem. In the last two to three decades, the applications have expanded to other fields, including neuroscience (e.g., Giorno et al 1997, Tuckwell 1988), biochemistry (e.g., Kruzynski \& Chelminiak 2002), biomedicine (Tuckwell \& Wan 2000), mathematical financing, image processing, and computer science.

The FPT approach is particularly advantageous in the scenarios where the system behavior is determined by the drift or diffusion of special functional parameters over time. The functional parameters refer to the technical parameters that can alter the reliability of the system elements (Pieper et al. 1997). Consider a system subject to time-dependent damage, the system fails when at least one of the functional parameters drifts away from a given region $(G)$ for the first time. Of course, the multiple functional parameters may be dependent with each other, which could cause significant complication. To some extent, FPT is a typical mathematical physics problem, where the interactions between 
two fields have been bi-directional historically. In mathematics, the most relevant tools for studying FPT are stochastic processes and partial differential equations (PDE), since both are the most natural ways to model diffusion processes, either stochastically or deterministically. However, only very limited number of FPT problems are analytically solvable with closed-form solutions. Currently, the FPT problem is exactly solvable only if the underlying stochastic process can be treated as a homogenous and diffusion process. Furthermore, known analytical solutions are limited to the one-dimensional case (Wu \& Fang 2008). In engineering science, numerical approaches, such as Monte Carlo Simulation and Response Surface Method can be employed to deal with complex nonlinearities and higher dimension issues (Labou 2003).

In computer science, the applications of FPT analysis include: estimation of bounds of success probability of Genetic algorithms (e.g., Aytug \& Koehler 2000, Pr'ugel-Bennett 2004, Yuen \& Cheung 2006), performance of artificial neural networks (e.g., Wang et al. 2008), ant colony optimization (e.g., Duan 2006), media access in wireless networks (e.g., Karamchandani et al. 2006, Ma et al. 2008), and software reliability. We will discuss the software reliability in a later section in more detail.

FPT and mean FPT are important characteristics of random walks in networks. Random walk in network is a very active research field and has been applied to fields such as transportation, communication, optimization search, etc (Argyrakisa \& Weiss 2006, Wang \& Pei 2008). Both single and multiple $(k)$ walkers in a network have been studied, and the latter is more complex but more realistic in describing natural phenomena, such as the transport of metabolite molecules through membrane channels (Argyrakisa \& Weiss 2006).

The FPT problem can be considered as the stochastic generalization of the shortest path problem (Wakuta 2000). The shortest path problem has been extended as a multi-objective decision problem by recasting it as a multi-cost optimization problem. Similarly, the FPT problem can be extended to consider multiple costs. The mean time to failure (MTTF) in reliability analysis may also be generalized as a FPT problem. However, mathematically, there is an essential difference between MTTF and FPT. The MTTF is a moment of the probability distribution that is used to describe the failure time random variable. The FPT is the time when a stochastic process crosses some threshold boundary, and the solution of the FPT problem is often in the form of a probability density function. More accurately, the solution of FPT is a family of functions, and it represents the evolution of PDF of FPT over time and space because it is the solution of PDE. The categorical form of FPT depends on boundary and initial conditions of the PDE.

Compared with other similar fields, FPT analysis in computer science is still relatively little known, especially in software reliability, network security, and network survivability. This lack of applications, and more importantly, what we believe is the huge potential of this research area, motivated us to write this article. Due to space limitation, this article is of abstract nature, and detailed studies will be reported in the future elsewhere. The remainder of the paper is organized as follows: Section 2 briefly introduces the mathematical analysis of the FPT problem; Section
3 analyzes two fields where FPT analysis has achieved significant success. This analysis is intended to inspire similar research in software reliability as well as network security and survivability. As it will become clear, the mathematical problems to be solved are essentially the same when abstracted into FPT problems. In Section 4, we discuss the potential of FPT analysis in software reliability as well as network security and survivability.

\section{FOKKER-PlANCK APPROACH TO FPT PROBLEM}

There are two major approaches for solving the FPT problem: analytic and simulation (numerical) approaches. The former depends on solving partial differential equations or stochastic differential equations. In particular, the Fokker-Planck diffusion equations are often used to describe FPT problems. The alternative simulation approach is often based on Monte Carlo simulation algorithms. In this subsection, we briefly outline an analytic procedure to obtain the FPT solution by solving the Fokker-Planck partial differential equation. This subsection draws on Ding and Rangarajan's (2004) presentation. Pieper et al (1997) and several others also performed similar studies. Comprehensive mathematical treatments of the Fokker-Planck approach can be found in monographs such as Risken (1989), Grimmet \& Stirzaker (1994), and Gardiner (1997).

Assuming a simple random walk with step $Y$ for every $\tau$ units of time described in the following form: $Y=l$ or $Y=-l$ with the equal probability of 0.5 . A new random variable $X_{n}$ is defined as:

$$
X_{n}=\sum_{i=1}^{n} Y_{i} \quad \text { with } X_{0}=0
$$

which represents the position of the random walker at time $t=n \tau$. Suppose that steps $Y_{i}$ are independent with each other. The mean squared displacement is denoted as

$$
\left\langle X_{n}^{2}\right\rangle=\sum_{i=1}^{n}\left\langle Y_{i}^{2}\right\rangle+\sum_{i \neq j}\left\langle Y_{i} Y_{j}\right\rangle=\sum_{i=1}^{n} l^{2}=n l^{2}=\frac{l^{2}}{\tau} t
$$

To simplify processing, one can take the diffusion limit: $\tau \rightarrow 0$, $l \rightarrow 0$ such that $l^{2} / t=2 D t$, where $\mathrm{D}$ is termed diffusion constant. With the simplification, one obtains:

$$
\left\langle X^{2}(t)\right\rangle=2 D t
$$

This is the equation that specifies Brownian motion (also known as the Wiener process). More generally, $X_{\mu}(t)$ is a Brownian motion with drift $\mu$ if

$$
X_{\mu}(t)=\mu t+X(t)
$$

In many applications, including the FPT problem, it is easier to deal with the PDF than to deal with the stochastic process itself. Furthermore, the PDF indeed contains sufficient information for the application in the case of the FPT problem. The PDF for the above described Brownian motion satisfies the well-known Fokker-Planck partial differential equation.

It can be verified that the Fokker-Planck PDE that describes the evolution of the PDF for Brownian motion is

$$
\frac{\partial}{\partial t} W(x, t)=D \frac{\partial^{2}}{\partial x^{2}} W(x, t)
$$


where $W(x, t)$ is the probability that the random walker is at location $x$ at time $t$. The boundary conditions for the equation are $W(x, t)=0$, at $x= \pm \infty$, and the initial condition is $W(x, 0)=\delta(x)$.

For Brownian motion with drift $a$, the Fokker-Planck PDE is

$$
\frac{\partial}{\partial t} W(x, t)=\mu \frac{\partial}{\partial x} W(x, t)+D \frac{\partial^{2}}{\partial x^{2}} W(x, t)
$$

According to the definition of FPT in Equation (1), the FPT problem with Brownian motion stochastic process is to find the solution of Fokker-Planck PDE represented with Equation (7) and the following boundary and initial conditions: $W(0, t)=W(\infty, t)=$ $0 ; \quad W(x, 0)=\delta(x-a)$, where $x=a$ is the new starting point of the Brownian motion. Ding \& Rangarajan (2004) demonstrated the PDF of the FPT as inverse Gaussian distribution:

$$
f(t)=\frac{a}{\sqrt{4 \pi D t^{3}}} \exp \left[-\frac{(a-\mu t)^{2}}{4 D t}\right], \quad a>0, \quad t>0
$$

When the PDF of the FPT is obtained, one can perform standard reliability analysis, similar to that performed using the Exponential or Weibull distribution models.

In the case of the continuous time one-dimensional random walk that is described with the following Langevin equation,

$$
\frac{d X}{d t}=\sum_{i=1}^{\infty} Y_{i} \delta\left(t-t_{i}\right)
$$

The random walker begins at $x=0$ at time $t_{0}=0$. Afterward, the walker waits at a given location $x_{i}$ for time $t_{i}-t_{i-1}$ before jumping $Y_{i}$ units. The jump size (y) may depend on the waiting time $u>0$, which itself could follow a probability distribution, such as

$$
\psi(u)=\int_{-\infty}^{+\infty} d y \phi(y, u)
$$

where $\phi(y, u)$ is the joint PDF of jump size $y$ and waiting time $u$. This continuous time random walk (CTRW) can be nonMarkovian. This is also known as Levy-type anomalous diffusion with zero drift. By using the fractional Fokker-Planck Equation (FFPE) approach and H-functions, Ding and Rangarajan (2004) derived the close-form PDF of the FPT. The form of the solution is very extensive and further introduction of the solution is beyond scope of this short article.

Fokker-Planck PDEs have very rich solutions and can be used to generate flexible PDFs by choosing various boundary and initial conditions. For example, in Equation (6), the boundary condition can be generalized to $x=a, b$, i.e., lower and upper bounds, which was mapped to the elastic barriers by Pieper et al. (1997) in structure reliability. The elastic barriers can be either absorbing or reflecting with some probability. In software reliability, the $x=a, b$ may be mapped to the discovery of bugs in "unit test" and "system test".

Another extension to Fokker-Planck PDE is to allow two drifting parameters $\mu_{1}, \mu_{2}$, or a vector of drifting parameters. This could be mapped to various levels of stress in structure reliability analysis. In software reliability, different drifting parameters may be mapped to different stages of testing, e.g., alpha or beta versions of software.
The FPT problem in higher dimensions is much more complex, and currently the closed-form PDFs are generally not available except for some special boundary conditions and initial conditions. For example, the transition probability density $p(x, t$, $y$ ) of the process $\vec{X}(t)$ can be solved from a Fokker-Planck PDE Equation in some special conditions. With multi-dimensional FPT problems, it is natural to consider elastic boundaries and different stress levels. The correlation and/or dependency between dimensions are of extreme significance, but they also cause significant complication. In general, the multidimensional FPT problem is a mixed blessing from the application perspective. On the one hand, the multidimensional or multivariate model is more realistic and flexible. On the other hand, the difficulty in obtaining analytic solutions and parameter estimation can make it impractical to apply. Ultimately, the curse of higher dimension may dictate that the parsimony principle should still be followed. Latest advances in Monte Carlo techniques such as Hiromoto (2007) and Blanchet \& Liu (2007) should be very helpful for numerical simulation approach. Hiromoto (2007) advanced a new iterative, boundary propagation scheme that uses successiveunder-relaxation (SUR) to improve the convergence in Monte Carlo simulation. Blanchet \& Liu (2007) proposed a simulation algorithm for estimating FPT probabilities in multidimensional random walks with $t$-distributed increments.

The difference between FPT and mean FPT (MFPT) is somewhat subtle. According to Kurzyn'ski \& Chełminiak (2003), by definition, the MFPT is obtained by observing the FPT in an ensemble of systems represented with a given stochastic process and averaging those FPTs demonstrated by members of the ensemble. An alternative is to observe some equivalent infinite process for a single system by assuming that the system will return to the initial state after crossing the threshold.

\section{Two APPLiCATION FiELDS: STRUCTURAL ANAlysis ANd Biochemical Process}

\subsection{Structure Analysis and Statistical Physics}

The reliability analysis of structures can be readily formulated as a FPT problem. Assuming a structure is exposed to stochastic dynamic loading characterized by a stochastic process (e.g., Brownian motion or Poisson process), $X(t)$, the first passage time (T) can be considered as the lifetime of the system (Labou 2003):

$$
T=\inf [t: g(X(t)) \leq 0, t \geq 0],
$$

where $g$ is called limit state function, and it can be used to define the boundary separating different system states. For example, $g(x)$ $=0$ can be used to separate reliable and unreliable states.

The probability of the first excursion (random walk) $P_{f}(t)$ within the time interval $t$ is defined by

$$
P_{f}(t)=P[T \leq t],
$$

Obviously, $1-P_{f}(t)$ is the reliability function. But the focus here is on specifying the evolution of failure probability with time.

Indeed, some of the most complex reliability analyses have been performed for structures (such as bridges and dams) that are subject to stochastic loading in civil and mechanical engineering. FPT is known as first-passage failure (or first excursion) in 
structure mechanics. It is a major failure model of mechanical or structural systems under random excitation, but its difficulty is also well-known in the theory of stochastic structural dynamics (Zhu et al. 2003). In such analysis, two failure mechanisms are often assumed: namely the first exceedance failure, and the failure due to damage accumulation (Pichler \& Pradlwarter 2008). In the first mechanism, a single crossing of the threshold causes failure. In the second mechanism, the failure is dependent on the number of crossings within a particular duration. Obviously, the first mechanism can be described with FPT problem. For example, Ching \& Beck (2007) studied the probability that any particular unobserved response of the structure exceeds a prescribed threshold by applying FPT approach, given incomplete output data from a structure excited by uncertain dynamic loading. Madhavan \& Prasad (2008) established the fatigue reliability degradation curve of offshore production and drilling platform with the FPT approach. The curve may be used for planning inservice inspection of offshore platforms.

\subsection{Application in Biochemistry}

The application of the FPT problem in the study of biochemical processes is particularly inspiring to computer scientists since the problem is transformed into a graph optimization problem, similar to the stochastic shortest path problem.

A challenge in the theory of biochemical processes becomes clear by the 1980s. Traditional chemical kinetics failed to explain the steady state kinetics of the biochemical process involving protein enzymes. One of the initial simple assumptions with conventional kinetics was the constant reaction rate, which is similar to the constant failure rate in a reliability function. Even with variable reaction rates, the ordinary differential equations were found insufficient. During the same period, the revealing of the transitions between a multitude of conformational sub-states in native proteins prompted the development of the stochastic dynamics theory. One of the stochastic dynamics theories is formulated as an FPT problem (Kruzynski \& Chelminiak 2002). This theory considers a biochemical reaction between two chemical species $M_{1}$ and $M_{2}$ involving protein enzyme, e.g., $M_{1} \leftrightarrow M_{2}$, as controlled and gated by the intramolecular dynamics of conformational transitions. The process could be abstracted as a graph model represented as a diagram (Fig. 1).

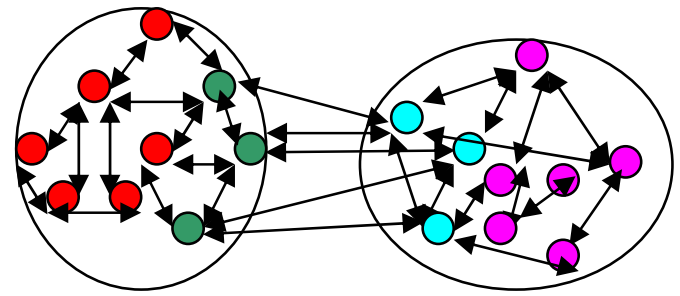

Fig. 1. The intramolecular dynamics underlying the uni-molecular reaction $M_{1} \leftrightarrow M_{2}$. Chemical states $M_{1}$ and $M_{2}$ of a macromolecule consist of many conformational sub-states (red and green circles, or pink and sky blue circles). The intramolecular dynamics is determined by purely stochastic conformational transitions (the arrows). The chemical reaction occurs through the transition between the sub-states. (Redraw after Kruzynski \& Chelminiak 2002).

The transition between $M_{1}$ and $M_{2}$ can be described with a system of stochastic differential equations (so-called Master equations):

$$
d p_{l}(t) / d t=\sum_{l^{\prime}}\left[w_{l l^{\prime}} p_{l^{\prime}}(t)-w_{l^{\prime} l} p_{l}(t)\right]
$$

The quantity $\mathrm{p}_{l}(t)$ is the probability of the macromolecule being in a conformational sub-state $l$ at time $t$. The coefficients $(w)$ are the transitional probabilities per unit time satisfying the detailed balance condition, which is too extensive to further explain in this short article but was fully documented in Kruzynski \& Chelminiak 2002. Obviously, there can be many sub-states within either $M_{1}$ or $M_{2}$, and there can be multiple chemical species $M_{i}$ in a biochemical process. Kruzynski \& Chelminiak (2002) adopted Hill's algorithm (Hill 1998) to compute the mean firstpassage time by 'summing up' the stochastic dynamics.

Now, if we envision the species $M_{1}$ and $M_{2}$ in Fig 1 as states of a network (e.g., healthy network vs. compromised network), the sub-states as the network nodes, the arrows within the 'species' as the network links, and the arrow between species as 'virtual transition,' then the original biochemical reaction can be transformed into a network security or survivability problem. That is, the transition between healthy and compromised network. The mean first passage time may be mapped to network lifetime.

\section{Application in Software Reliability, NETWORK SECURITY AND SURVIVABILITY}

\subsection{Software Reliability}

Software reliability uses stochastic modeling extensively, and it is estimated that over 200 software reliability models have been proposed (Rinsaka et al. 2006). According to Rinsaka et al. (2006), the classical and perhaps the most important software reliability model is the non-homogeneous Poisson process (NHPP) model, which characterizes the number of faults detected in the software testing phase and can be used to predict software reliability. The traditional approach in this venue of software reliability has been focused on studying the stochastic process itself. However, the direct approach to stochastic process can be very complex, and analytical solutions may be difficult to obtain. We conjecture that this difficulty may explain why the NHPP has been dominantly used in software reliability, since the Poisson process is the simplest stochastic process, although its nonhomogenous version brings in more flexibility and power. An exception to the above general trend is the study by Rinsaka \& Dohi (2004). They assumed that the software fault detection process follows Markovian birth process, and they adopted an FPT model available in literature to obtain the optimal software release time. What we emphasize here is that FPT analysis can be used as a general approach for software reliability modeling, and it is expected that many of the existing reliability models are the special cases of the FPT analysis.

Another dominant research area in software reliability is to study the relationship between software quality (reliability) and various software metrics. In general, there are four categories of software metrics: product metrics, development metrics, deployment and usage metrics, and software-hardware configurations metrics (Rinsaka et al. 2006). It is hoped that the analysis of the relationship between software reliability and metrics will provide better predictions of software reliability. In this venue, it is obvious that survival analysis can play a critical role. Currently, 
only univariate survival analysis is applied occasionally, and multivariate survival analysis, even competing risks analysis, can provide more powerful and flexible approaches (Ma 2008). Of course, the above two areas (stochastic modeling and metrics) can be integrated (e.g., Shibata et al. 2006). In addition, survival analysis and FPT approach can also be integrated to study reliability. Zhao \& Elsayed (2004) already reported such an example. They integrated FPT with competing risks analysis for studying system performance degradation. In their study, they treated performance degradation as a Brownian motion process, and then, the FPT to a boundary can be considered a soft failure. They described hard (catastrophic) failure with the traditional Weibull distribution. Consequently, both hard failure and soft failure are competing risks, and competing risks analysis is used to naturally integrate two modeling threads. What we suggest here is that survival analysis can be integrated with the FPT approach to take advantages from both approaches, with FPT focusing on failure mechanisms with its analytic solutions and survival analysis focusing on modeling of metrics data.

In software reliability, multidimensional FPT can be very useful. For example, in a client-server software system, the functionality of the system depends on both client and server. The reliability of the client-server system may be formulated as a two-dimensional FPT problem or a three-dimensional FTP problem (with network linking as the third dimension). In a distributed system, the reliability of local vs. remote nodes may also be formulated as a two-dimensional random walk.

\subsection{Network Security and Survivability}

Nwankpa (1992) suggested the connection between FPT and security in the context of security of power systems. This was prior to that when network security became a major issue, and his work seems to be the first application of FPT to security field. $\mathrm{He}$ proposed to use mean FPT as the dynamic security index for power system, which measures the average time a power system will eventually take to reach its dynamic security limits when affected by a contingency. Nwankpa's (1992) work was formulated more like a survivability analysis. Of course, the paradigm of survivability was advanced a few years after his work. We consider Nwankpa's (1992) arguments are valid for both network security and survivability.

In subsection 3.2, we proposed the analogical study of biochemical process with network security. Besides, the FPT approach may be used for modeling other security events, such as denial of service attack, or the behaviors of hackers or terrorists. In the case of denial of service, the FPT can be mapped to the point when the network ceases to provide useful service to its legitimate users. Similarly, FPT may be used to model the first

\section{REFERENCES}

[1] Argyrakisa, P. \& G. H. Weiss. A first-passage time problem for many random walkers. Physica A 363 (2006) 343-347.

[2] Aytug H., G. J. Koehler. 2000. New stopping criterion for genetic algorithms. European Journal of Operational Research 126:662-674. strike time of a hacker by assuming his behaviors conform to some stochastic processes.

Similar to the study of software reliability discussed in Subsection 4.1, we suggest that FTP and survival analysis can be integrated to model network security and survivability. For example, FPT can be introduced into the three-layer survivability analysis and dynamic hybrid fault models (Ma 2008, and Ma \& Krings 2008), either as an analytical alternative to survival analysis or as being integrated with survival analysis. The advantages from the integration should be more flexible and realistic modeling of reliability, fault tolerance, security and survivability in a comprehensive manner. Certainly, the introduction of FPT into the three-layer survivability analysis, dynamic hybrid fault models, or even the standard-alone applications to software reliability, network security and survivability is a complex procedure. In the following Figure 2, we summarize the major steps in applying FPT analysis to reliability, security and survivability in a diagram.

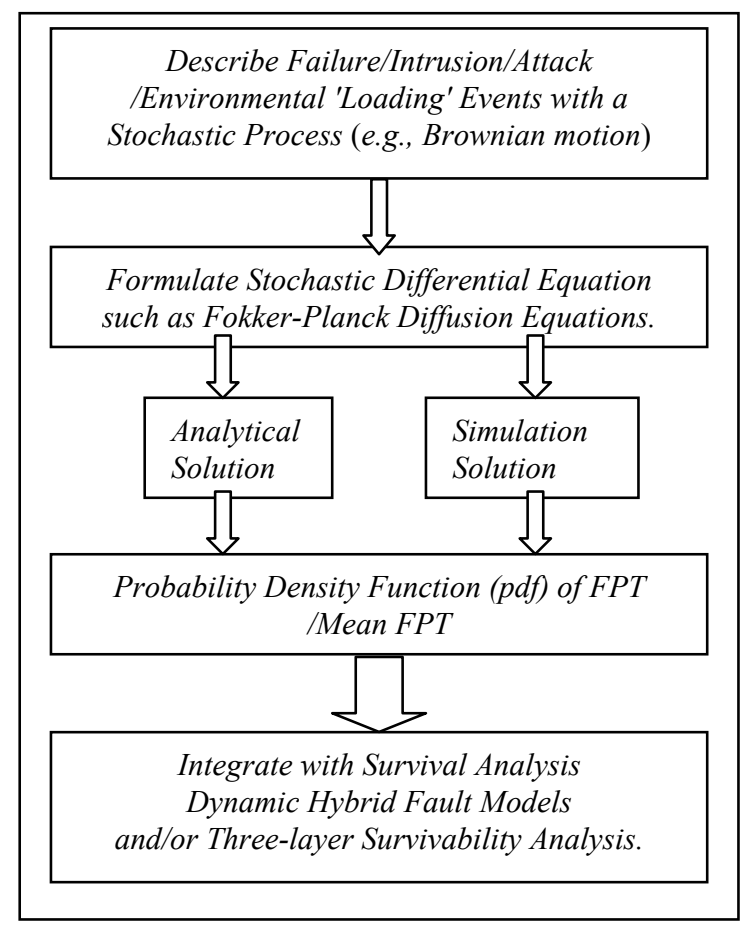

Figure 2. A diagram showing the major steps involved in the application of FPT approach to software reliability, network security and survivability.

[3] Bachelier, L. 1900. Annales des Sciences de l'Ecole Superieure 17, 21.

[4] Blanchet, J. H., J. Liu. 2007. Rare-event simulation for a multidimensional random walk with t-distributed increment. Proc. of the 2007 IEEE Winter Simulation Conference.

[5] Ching, J., J. L. Beck. 2007. Real-time reliability estimation for serviceability limit states in structures with uncertain dynamic excitation and incomplete output data. Probabilistic Engineering Mechanics 22 (2007) 50-62 
[6] Ding, M. \& G. Rangarajan. 2004. First Passage Time Problem: A Fokker-Planck Approach. in "New Directions in Statistical Physics". ed by L. T. Wille. Springer. pp31-46.

[7] Duan, H., D. Wang, X. Yu. 2006. Markov Chains and Martingale Theory Based Convergence Proof of Ant Colony Algorithm and Its Simulation Platform. Proc. of the 6th World Cong. on Intelligent Control and Automation, June 21-23, 2006, Dalian, China

[8] Gardiner, C. W. 1997. Handbook of Stochastic Methods. Springer.

[9] Giorno, V. A. G. Nobile, L. M. Ricciardib. 1997. BioSystems 40: $65-74$

[10] Grimmet, G. R. \& D. R. Stirzaker. 1994. Probability and Random Process. Oxford University Press, Oxford.

[11] Hill, T. L. 1989. Free energy transduction and biological cycle kinetics. Springer, New York.

[12] Hiromoto, R. E. 2007. An Iterative Monte Carlo Scheme with Relaxation. IEEE International Workshop on Intelligent Data Acquisition and Advanced Computing Systems: Technology and Applications. 6-8 September 2007.

[13] Karamchandani, N., D. Manjunath, D. Yogeshwaran and S. K. Iyer. 2006. Evolving Random Geometric Graph Models for Mobile Wireless Networks. 2006 4th International Symposium on Modeling and Optimization in Mobile, Ad Hoc and Wireless Networks.

[14] Kruzynski, M. \& P. Chelminiak. 2002. Mean first-passage time in the stochastic theory of biochemical processes. Journal of Statistical Physics. 110:137-181.

[15] Kulkarni, V. G. E. Tzenova. 2002. Mean first passage times in fluid queues. Operations Research Letters 30: 308-318.

[16] Labou, M. 2003. Solution of the First Passage Problem by advanced Monte Carlo Simulation Technique. Strength of Materials, 35(6), 2003

[17] Ma, Z., Z. Cao, W. Chen. 2008. A Fair Opportunistic Spectrum Access (FOSA) Scheme in Distributed Cognitive Radio Networks. 2008 IEEE Int. Conf. Comm.

[18] Ma, Z. S. 2008. New approaches to reliability and survivability with survival analysis, dynamic hybrid fault models, and evolutionary game theory. $\mathrm{PhD}$ Dissertation, $177 \mathrm{pp}$, University of Idaho, Moscow, ID, USA.

[19] Ma, Z. S. \& A. W. Krings. 2008a Multivariate Survival Analysis (I): Shared Frailty Approaches to Reliability and Dependence Modeling, Proc.29th IEEE-AIAA Aerospace Conference, March 1-8, 2008, Big Sky, MT. 21 Pages.

[20] Ma, Z. S. \& A. W. Krings. 2008b. Dynamic hybrid fault models and their applications to wireless sensor networks. The 11-th ACM/IEEE International Symposium on Modeling, Analysis and Simulation of Wireless and Mobile Systems. ACM MSWiM 2008. 9 pages.

[21] Madhavan P. \& M. Prasad. 2008. Fatigue reliability analysis of fixed offshore structures: A first passage problem approach. Journal of Zhejiang University Science A. 7(11):1839-1845

[22] Nwankpa, C. O. 1992. The Search for An On-Line Dynamic Security Index. IEEE International Conference on Systems, Man and Cybernetics. Digital Object Identifier: 10.1109/ICSMC.1992.271644.
[23] Pichler, L, Pradlwarter H. J. 2008. Evolution of probability densities in the phase space for reliability analysis of nonlinear structures. doi:10.1016/j.strusafe.2008.09.002

[24] Pieper, V., Domine, M., Kurth, P. 1997. Level crossing problems and drift reliability. Mathematical Methods of Operations Research. 45:347-354.

[25] Pr'ugel-Bennett, A. 2004. When a genetic algorithm outperforms hill-climbing. Theoretical Computer Science 320:135-153

[26] Rangarajan, G., M. Ding, M. Z. 2000. First passage time distribution for anomalous diffusion. Physics Letters A 273:322-330

[27] Revelli, J. A. C. E. Budde, H. S. Wioa. 2006. Diffusion in fluctuating media: first passage time problem. Physics Letters A. 306 (2002) 104-109.

[28] Rinsaka, K and T. Dohi. 2004. Who Solved the Optimal Software Release Problems Based on Markovian Software Reliability Model? Proc. of the 47th IEEE International Midwest Symposium on Circuits and Systems.

[29] Rinsaka, K., K. Shibata, T. Dohi. 2006. Proportional Intensity-Based Software Reliability Modeling with TimeDependent Metrics. Proceedings of the 30th Annual Int. Computer Software and Applications Conf. (COMPSAC'06).

[30] Risken, K. 1989. The Fokker-Planck Equation. Springer.

[31] Shibata, K. K. Rinsaka, T. Dohi. 2006. Metrics-Based Software Reliability Models Using Non-homogeneous Poisson Processes. 17th International Symposium on Software Reliability Engineering (ISSRE'06)

[32] Tuckwell, H. C. 1988. Introduction to theoretical neurobiology. Cambridge Univ. Press.

[33] Tuckwell, H. C. and F. Y. M. Wan. 2000. First Passage Time to Detection in Stochastic Population Dynamical Models for HIV-1. Applied Mathematics Letters 13 (2000) 79-83.

[34] Wakuta, K. 2000. A First Passage Problem with multiple costs. Mathematical Methods of Operations Research. 51:419-32

[35] Wang, S. P., W. J. Pei, Z-Y. He. 2008. Random Walks On the Neural Network of C.elegans. IEEE Int. Conference Neural Networks \& Signal Processing.

[36] Wang, S. P. W. J. Pei. 2008. First passage time of multiple Brownian particles on networks with applications. Physica A 387: 4699-4708

[37] Wu, Y. and W. Fang. 2008. Stochastic averaging method for estimating first-passage statistics of stochastically excited Duffing-Rayleigh-Mathieu system. Acta Mech Sin. 24:575582

[38] Yuen, S. Y. and B. K. S. Cheung. 2006. Bounds for Probability of Success of Classical Genetic Algorithm Based on Hamming Distance. IEEE Trans. Evol. Comp. 10(1):1-18.

[39] Zhao, W. \& E.A. Elsayed. 2004. An Accelerated Life Testing Model Involving Performance Degradation. IEEE RAMS 2004.

[40] Zhua, W. Q. Z.L. Huang, M. L. Deng. 2003. First-passage failure and its feedback minimization of quasipartially integrable Hamiltonian systems. International Journal of Non-Linear Mechanics 38:1133-1148 


\section{Introduction of First Passage Time (FPT) Analysis for Software Reliability and Network Security}

Sam Ma Axel Krings

(University of Idaho)

Richard Millar

(Naval Postgraduate School)

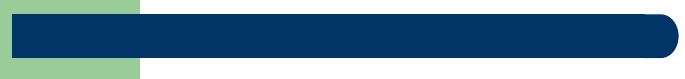

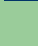

\section{Outline}

- Introduction

- Fokker-Planck Approach to FPT Problem

- Two Application Examples: Structural Analysis and Biochemical Process

- Potential for Software Reliability, Network Security and Survivability Research

- Summary 


\section{What is First Passage Time (FPT) Problem?}

- Informally, The FPT is the time (T) when a stochastic process crosses some threshold boundary.

- Mathematically, given a stochastic process $X(t)$ with $X(0)=0$, the FPT $T$ to the point $x=a>0$ is specified with:

$$
T=\inf \{t: X(t)=a\} .
$$

- The FPT problem usually refers to finding the probability density function $(P D F)$ of $T$.

\section{Applications of FPT Problem}

- $\quad$ Study of FPT as early as 1900.

- Probability theory

- Statistical Physics

- Civil and Mechanical Engineering

- Hydrology

- Chemical Physics

- $\quad$ Financial Engineering

- Image Processing

- Neuroscience

- Biochemistry

- Computer Science 


\section{Applications of FPT Problem in Computer Science}

- Bounds of success probability of Genetic algorithms, and Ant Colony optimization.

- Channel access in wireless networks.

- Stochastic generalization of the shortest path problem.

- Software reliability modeling.

\section{FPT Problem and Mean Time to Failure (MTTF)}

- The mean time to failure (MTTF) in reliability analysis may also be generalized as a FPT problem. However, there is an essential difference between MTTF and FPT.

- The MTTF is a moment of the probability distribution that describe the failure time random variable.

- The FPT is the time (T) when a stochastic process crosses some threshold boundary, and the solution of the FPT is in the form of a probability density function (PDF).

- More accurately, the solution of FPT is a family of functions, and it represents the evolution of PDF of FPT over time and space because it is the solution of Partial Differential Equation (PDE). The categorical form of FPT depends on the boundary and initial conditions of the PDE. 
FPT Problem and Random Walks on Networks

- FPT is a very important characteristic of random walks on network (graph). Single walker can be characterized with FPT. $k$-walkers can be characterized with Mean FPT.

- Random walks on networks have wide applications, e.g., optimization problem, transportation of metabolites between cell membrane.

- Similarly, FPT problem exists in Percolation systems and Random Cluster. Therefore, FPT problem is important for the study of critical phenomenon, including the connectivity in communication

7 networks.

\section{Outline}

- Introduction

- Fokker-Planck Approach to FPT Problem

- Two Application Examples: Structural Analysis and Biochemical Process

- Potential for Software Reliability, Network Security and Survivability Research

- Summary 


\section{Two Approaches for Solving the}

FPT Problem

Two major approaches for solving FPT problem:

- Simulation (often based on Monte Carlo simulation algorithms) approaches.

- Analytic Approaches: depends on solving partial differential equations. In particular, the FokkerPlanck diffusion equations are often used to describe FPT problems.

\section{Fokker-Planck Approach to FPT Problem.}

- Step 1. Find the stochastic process that describes the problem (govern the process), e.g., the dynamic loading a bridge experiences, the usage pattern of a software feature, the activities of hackers, etc, may follow a stochastic process (e.g., Brownian motion)

- Step 2. Formulate the Fokker-Planck partial differential equation and set boundary and initial conditions.

- Step 3. Solve the Fokker-Planck equation and the 10 solutions are the PDF of the FPT. 


\section{Fokker-Planck Approach to FPT: an example with Brownian motion}

- Step 1. Assume a process follows Brownian motion, $X(t)$. The direct analysis of the process is usually very difficult.

- Step 2. The corresponding F-P equation is:

$$
\frac{\partial}{\partial t} W(x, t)=\mu \frac{\partial}{\partial x} W(x, t)+D \frac{\partial^{2}}{\partial x^{2}} W(x, t)
$$

- Step 3. The solution or the PDF of the FPT is:

$$
f(t)=\frac{a}{\sqrt{4 \pi D t^{3}}} \exp \left[-\frac{(a-\mu t)^{2}}{4 D t}\right], \quad a>0, \quad t>0
$$

\section{Outline}

- Introduction

- Fokker-Planck Approach to FPT Problem

- Two Application Examples: Structural Analysis and Biochemical Process

- Potential for Software Reliability, Network Security and Survivability Research

- Summary 
(1) Structural Analysis in Mechanical and Civil Engineering

- Assuming a structure is exposed to stochastic dynamic loading characterized by a stochastic process (e.g., Brownian motion or Poisson process), $X(\mathrm{t})$, the first passage time $(T)$ can be considered as the lifetime of the system (Labou 2003):

$$
T=\inf [t: g(X(t)) \leq 0, t \geq 0],
$$

$\mathrm{g}$ is limit state function, and it can be used to define the boundary separating different system states, e.g., $g(x)=0$ can be used to separate reliable and unreliable states.

(1) Structural Analysis in Mechanical and Civil Engineering

- The probability of first passage failure (excursion) $P_{f}(t)$ within the time interval $t$ is defined by

$$
P_{f}(t)=P[T \leq t]
$$

- Obviously, $1-P_{f}(t)$ is the reliability function. But the focus here is on specifying the evolution of failure probability with time. 
(2) Biochemical Process: It is transformed into a graph optimization problem, similar to the stochastic shortest path problem.

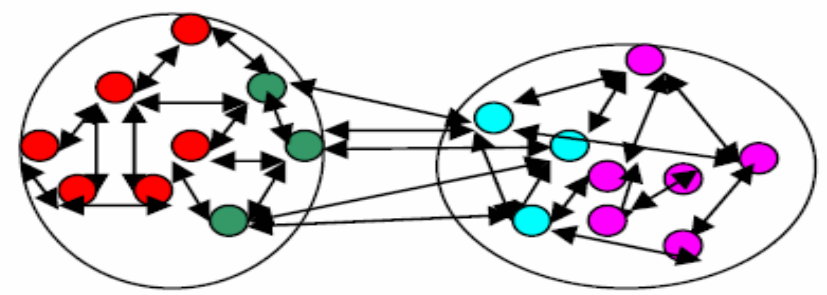

Fig. 1. The intermolecular dynamics underlying the uni-molecular reaction $M_{1} \leftrightarrow M_{2}$. Chemical states $M_{1}$ and $M_{2}$ of a macromolecule consist of many conformational sub-states (red and green circles, or pink and sky blue circles). The intramolecular dynamics is determined by purely stochastic conformational transitions (the arrows). The chemical reaction occurs through the transition between the sub-states. (Redraw after Kruzynski \& Chelminiak 2002).

\section{Outline}

- Introduction

- Fokker-Planck Approach to FPT Problem

- Two Application Examples: Structural Analysis and Biochemical Process

- Potential for Software Reliability, Network Security and Survivability Research

- Summary 


\section{Software Reliability: First venue -Stochastic Process Modeling.}

- Rinsaka et al (2006): It is estimated that over 200 software reliability models have been proposed with this approach

- The classical and perhaps the most important model is the non-homogeneous Poisson process (NHPP) model, which characterizes the number of faults detected in the software testing phase and can be used to predict software reliability.

- The traditional approach in software reliability has been focused on studying the stochastic process itself. However, the direct approach to stochastic process can be very complex, and analytical solutions may be difficult to obtain.

- FPT analysis with focus on PDF allows the study of more complex stochastic processes such as Brownian motion.

\section{Software Reliability: Second Venue}

-Relationship between software reliability \& metrics.

- Various statistical modeling approaches have been used.

- We believe that Survival Analysis should be a major approach. However, it seems largely unknown in software reliability field.

- FPT can help develop new theoretical models of survival analysis, Hougaard (2000) $d Z_{t}=a(t) d t-b(t) Z_{t} d t+c d W_{t}, \quad Y_{t}=Z_{t}^{2}$ Population hazard: $\omega(t)=\lambda(t)\left[m(t)^{2}+\sigma(t)^{2}\right]$

- Multidimensional FPT: e.g., in a client-server software system. The reliability of the client-server system may be formulated as a twodimensional FPT problem or a three-dimensional FTP problem (with network linking failure). In a distributed system, the reliability of local vs. remote nodes may be formulated as a two-dimensional problem. 


\section{Network Security and Survivability}

- Nwankpa (1992) suggested the connection between FPT and security of power systems. He proposed to use mean FPT as the dynamic security index for power system, which measures the average time a power system will eventually take to reach its dynamic security limits when affected by a contingency.

- Prior to that when computer network security became a major issue. His work is more like survivability we talk today.

- Computer networks are much more complex. More complex modeling such as developed in biochemistry application may be needed.

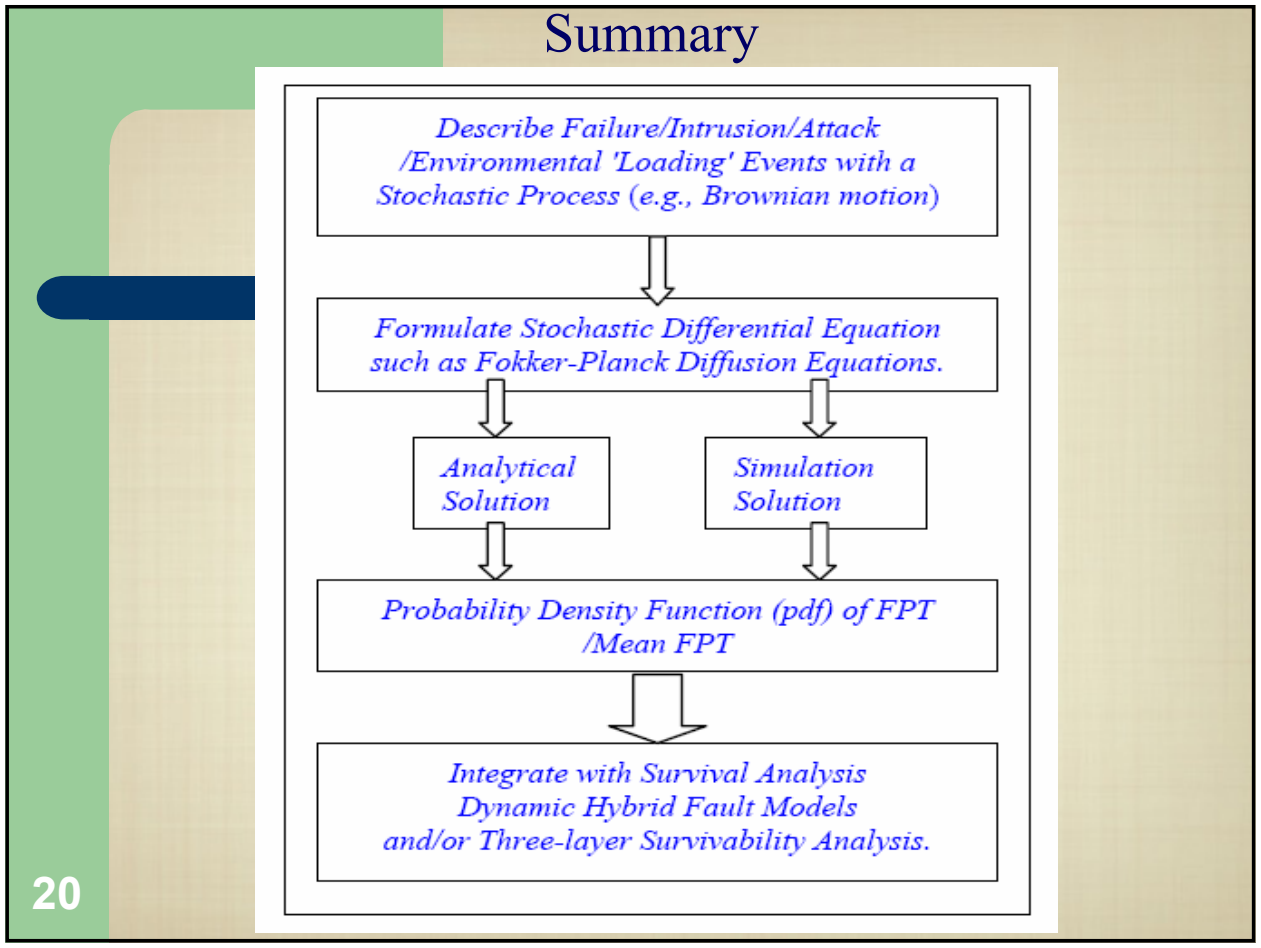

